\title{
SHORTER COMMUNICATIONS
}

\section{ONSET OF CONVECTION NEAR A SUDDENLY HEATED HORIZONTAL WIRE}

\author{
C. M. VEST and M. L. LAWSON \\ Department of Mechanical Engineering, The University of Michigan, Ann Arbor, Michigan 48104, U.S.A.
}

(Revised 30 September 1971 and in revised form 5 November 1971)

\section{INTRODUCTION}

THIS note deals with the early transient processes which occur in a fluid surrounding a thin horizontal wire which is suddenly heated by passing an electrical current through it. Observation of the resulting temperature field with a Mach-Zehnder interferometer shows that the isotherms in a plane normal to the wire are initially concentric circles. This indicates that heat transfer is by conduction only. After a period of time the isotherms become asymmetrical as those above the wire are convected upward while those below the wire remain nearly stationary. The upward transport accelerates until finally a heated "cap" breaks away and rises to form the familiar mushroom-shaped steady state plume. In this study attention is focused on the "delay time", $t^{*}$, between the application of current to the wire and the beginning of observable convection. It is hypothesized that the onset of convection is a manifestation of instability, at least in the sense that it occurs at a specific critical value of an appropriately defined Rayleigh number. A simple quasisteady analysis predicts the delay time as a function of the rate of energy dissipation in the wire and the transport properties of the fluid. The delay time was also determined experimentally for a wide range of heat transfer rates. The experimental data is in substantial agreement with the theoretical prediction.

\section{ANALYSES}

Experimental observation of the temperature field surrounding a suddenly-heated thin horizontal wire indicates that for small values of time there is no perceptible convection; hence, if the heat capacity of the wire is neglected, the temperature field $\theta(r, t)$ in the fluid is that due to conduction from a line source of strength $q^{\prime}$ in an infinite medium which was initially isothermal at temperature $\theta=0$. The exact solution of this problem in terms of an exponential integral is classical [1]: however, for present purposes it is preferable to utilize a simple approximation to this solution, namely

$$
\left[\theta=\frac{q^{\prime}}{2 \pi k}\left(\frac{r}{\delta}\right)^{2}-\frac{r}{\delta}-\ln \frac{r}{\delta}\right] .
$$

Here $k$ is the thermal conductivity of the fluid and $\delta$ is the instantaneous radial "penetration depth" at which the approximate temperature field and its first derivative vanish. Upon solving the unstcady conduction cquation on an integral basis this penetration depth is found to be

$$
\delta=\sqrt{ }(6 \alpha t) .
$$

where $\alpha$ is the thermal diffusivity. It is now hypothesized that the fluid directly above the wire becomes locally unstable at some critical Rayleigh number, and that convective transport thereafter becomes significant. Since the medium is infinite and since the radially symmetric temperature field gives rise to density gradients normal to the gravity vector, the fluid is always globally unstable: however, disturbance growth rates may be quite small until some minimum temperature gradient is attained. If so, a local quasi-stcady stability analysis of the fluid dircetly above the wire is appropriate. To pursue this argument it was assumed that the critical Rayleigh number might be associated with that of the classical Bénard problem of a fluid layer heated from below. This approach was taken because the initial motion of the isotherms was observed to be strongest directly above the wire, where the temperature and density gradients are parallel to the gravity vector. The Rayleigh number, $R=g \gamma \beta \delta^{4} / \alpha \nu$, was defined in terms of the gradient

$$
\beta=\frac{5 q^{\prime}}{6 \pi k \delta}
$$

which corresponds to the linear temperature distribution whose integral from $r=0$ to $r=\delta$ is the same as that of the distribution (1). Here $\gamma$ is the thermal expansion coefficient, $\alpha$ is the thermal diffusivity and $v$ is the kinematic viscosity. The critical Rayleigh number was taken to be $R=1100$. corresponding to the Bénard problem with rigid lower and "free" upper boundaries [2]. When equations (2) and (3) are substituted into the definition of $R$, which is then equated to 1100 , the resulting expression for the "delay time" at which significant convection is initiated is

$$
\left(\frac{g \gamma \sqrt{ } \alpha}{k v}\right)^{\frac{3}{3}} t^{*}=43\left(q^{\prime}\right)^{-\frac{3}{3}}
$$

The argument used to derive the expression (4) for the delay time is similar to that used by Howard [3] to estimate 
mean temperature profiles in high Rayleigh number convection in terms of an analogous delay time required for the formation and breakaway of thermals from the conductive sublayer. It is also interesting to note that two strictly empirical relations given by Ostroumov [4] [his equations (1) and (2)] can be combined to suggest that $t^{*}=$ const. $\left(q^{\prime}\right)^{-0 \cdot 6}$.

\section{EXPERIMENT}

The transient temperature field in the fluid surrounding a suddenly heated thin horizontal wire was observed using a precision Mach Zehnder interferometer with a $\mathrm{He} \mathrm{Nc}$ laser light source. The test section in which the heated wire was housed was a steel chamber $(48 \mathrm{~cm} \times 43 \mathrm{~cm} \times 38 \mathrm{~cm})$ with optically-flat windows. The optics and test section were mounted on a massive platform supported by inllated inner tubes in order to isolate them from building vibrations. The $0.203 \mathrm{~mm}$ dia, tungsten wire was mounted on a support such that its length and orientation could be varied. It was energized by two $12 \mathrm{~V}$ storage batteries connected in parallel. The applied voltage was adjusted by a bank of resistors and was measured with a calibrated storage oscilloscope.

The wire was placed in the test section and carefully adjusted to be horizontal and in proper alignment with the optical axis of the interferometer. The entire system was left undisturbed for a few hours preceding each run so that the fluid in the test section became quiescent. Current was then applied to the wire and the resulting transient pattern of isotherms (interference fringes) was recorded with a $16-\mathrm{mm}$ movie camera at a speed of 32 or $64 \mathrm{frames} / \mathrm{s}$. The time at which current was applied was marked by the firing of a flash lamp, and the camera speed was calibrated.

Figure 1 shows a typical sequence of interferograms rccorded after application of current to the wire, which was perpendicular to the plane of this figure. In Fig. 1(a) the temperature field is symmetric about the wire, i.e. heat transfer is essentially by conduction alone. Figure (b) shows the asymmetry of the temperature field at a time slightly greater than $t^{*}$. At still greater times convection is an important mode of energy transport and the temperature field grows as shown in Figs. 1(c) and 1(d). The position of the outermost isotherm as a function of time was measured by using a projecting microscope to analyze the movie film from which the sequence of Fig. I was taken. Figure 2 is a plot of the position of the outer isotherm directly above and directly below the wire as a function of time. The early symmetrical growth followed by upward convection above the wire is evident. These two ligures deal with experiments in water: those in air were qualitatively the same.

The delay time, $t^{*}$, was determined by examining the film frame-by-frame to locate the first time at which an appreciable lack of symmetry in the outermost isotherm was observed. This determination was generally unambiguous to within about three frames. 28 acceptable runs were made using water with heat transfer rates varying from 4 to 530 $\mathrm{Btu} / \mathrm{htt}$. The observed delay times varied from 11.2 to $0.63 \mathrm{~s}$. In air. 14 runs were made over a heat flux range of 9 to $52 \mathrm{Btu} / \mathrm{ht}$, and observed delay times varied from 0.25 to $0.094 \mathrm{~s}$. This data is summarized in Fig. 3. The solid line in

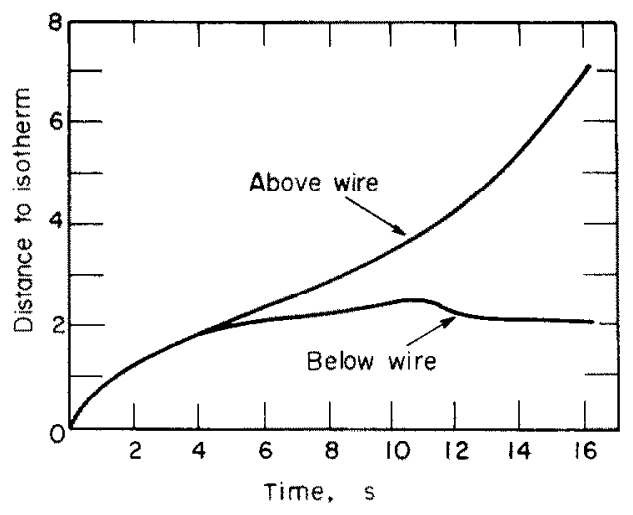

FIG. 2. Growth of the distance from the wire to the outer isotherm directly above and directly below the wire for $q^{\prime}=11 \mathrm{Btu} / \mathrm{hft}$ in water. Distance units are arbitrary.

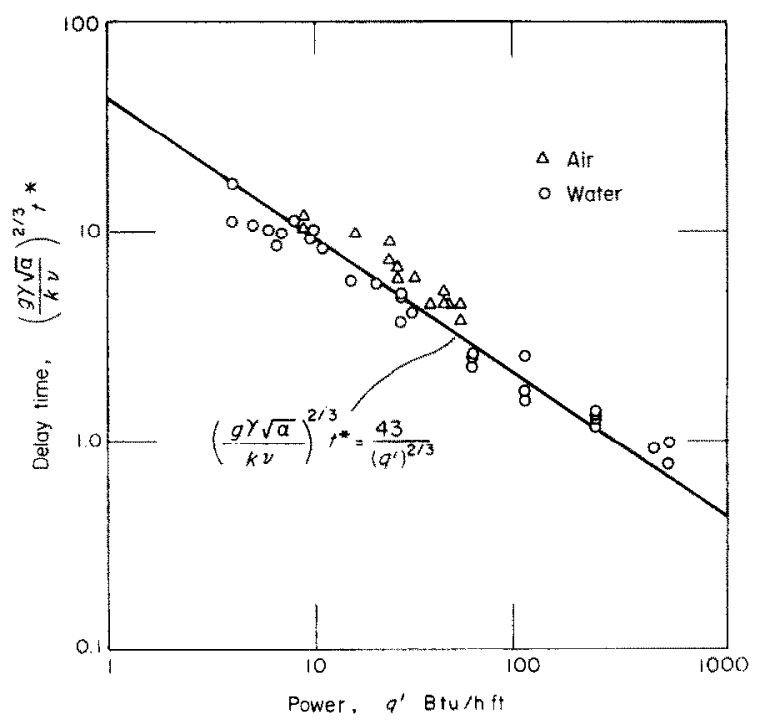

Fis 3. Delay times in air and water.

this figure is a plot of the theoretical delay time given by equation (4).

The experimentally determined values of delay time in water correlate reasonably well with those predicted by the approximate analysis presented above. The data for air. while following the general trend of equation (4). falls consistently above the line. This may be explained in part by the neglect of the thermal capacity of the wire in the analysis.

Observations were also made in water with the horizontal wire mounted perpendicular to the optical axis of the interferometer. Convection cells were indeed observed above the wire during the rise of the layer of heated fluid. A clear indication of the existence of such convection cells in Freon 

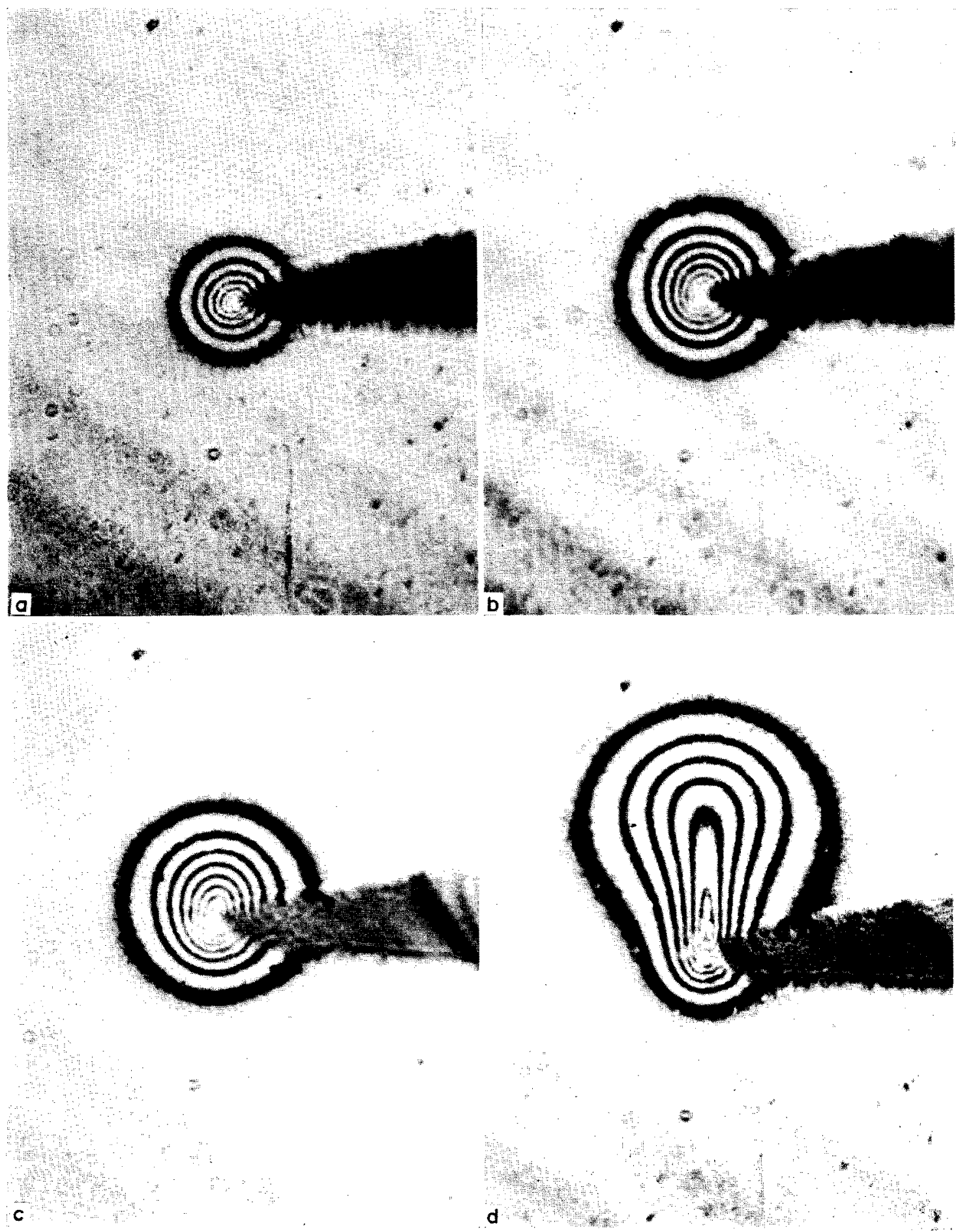

Frg. 1. Developing temperature field in water for $q^{\prime}=11 \mathrm{Btu} / \mathrm{hft}$.

(a) $t=3.91 \mathrm{~s}$

(c) $t=9 \cdot 31 \mathrm{~s}$

(b) $t=8 \cdot 13 \mathrm{~s}$

(d) $t=16.7 \mathrm{~s}$

H. M. 
above a suddenly heated wire can be seen in the photographs on p. 347 of [5]. These observations strengthen the assumption that $R \sim 1100$ at $t^{*}$. The sensitivity of the interferometric method with the fluids used in the present study was insufficient to permit a detailed investigation of the structure of these cells.

\section{CONCLUSIONS}

Experimental measurements of the delay time during which heat transfer from a suddenly heated fine horizontal wire to a tluid is essentially by conduction agree rather well with those predicted by equation (4). This correlation suggests that the onset of significant convection above a heated horizontal wire is associated with a fluid instability in the sense that there is a critical value of an appropriately defined Rayleigh number below which disturbances grow slowly with respect to the characteristic thermal diffusion time of the fluid. Above this critical value disturbances grow rapidly and initiate significant convection. The expression (4) for the delay time may also be useful as a guide in the design of experiments to determine thermal diffusivities by transient response measurements, and for the interpretation of experimental data regarding onset of boiling and other heat-transfer phenomena near suddenly heated wires.

\section{ACKNOWLEDGEMENTS}

The authors wish to express their appreciation for the support of the Horace H. Rackham School of Graduate Studies and to D. W. Sweeney and A. R. Brunsvold for their assistance in construction of the optical systems and test section.

\section{REFERENCES}

1. H. S. Carslaw and J. C. Jaeger. Conduction of Heat in Solids, 2nd ed. Oxford University Press, London (1959).

2. S. ChandRaseKhar, Hydrodynamic and Hydromagnetic Stability, p. 43. Oxford University Press, London (1961).

3. L. N. Howard, Convection at high Rayleigh number, Proc. 11th Intern'l. Congress of Appl. Mech.. Munich, pp. 1109-1115 (1964).

4. G. A. Ostroumov. Unsteady heat convection near a horizontal cylinder, J. Tech. Phys. USSR 1, 2627-2641 (1956).

5. W. Hauf and U. Grigull, Optical methods in heat transfer, Advances in Heat Transfer, edited by J. P. HarTNETT and T. F. IRVine, JR., Vol. 6. pp. 134-366. Academic Press, New York (1970).

\title{
TO GEOMETRIZED THEORY OF HYPERBOLIC HEAT CONDUCTION EQUATION
}

\author{
V. L. KOLPASHCHIKOV and A. A. BARANOV \\ Heat and Mass Transfer Institute, Byelorussian Academy of Sciences, Minsk, U.S.S.R.
}

(Received 2 Decemher 1971)

1. IT IS well known that a linear parabolic heat-conduction equation leads to the paradox of infinite velocity of propagation of heat disturbances. All attempts to solve this paradox have revealed the necessity to consider a hyperbolic heattransfer equation. A number of papers $[1-5]$ are concerned with the derivation and substantiation of this equation. A. V. Luikov [1,2] has found that an equation of the form

$$
c_{\gamma} \frac{\partial T}{\partial t}+\frac{\lambda}{w^{2}} \frac{\partial^{2} T}{\partial t^{2}}=\lambda \nabla^{2} T+p
$$

holds for capillary-porous systems, if a tinite velocity of heat and mass propagation is assumed. In equation (1) $w$ is the heat propagation velocity $w=\sqrt{ }\left(\lambda / c_{\gamma} \tau\right), \tau$ is the relaxation time, $p$ is the heat source function, $\lambda$ is the thermal conductivity, $c_{\gamma}$ is the volumetric heat capacity.

In case of small $c_{y}$ and large mean free molecular path lengths the first term in the right hand side of equation (1) is small compared to the other terms and may therefore be omitted. The wave form of the heat-conduction equation is obtained as a result

$$
\frac{\partial^{2} T}{\partial t^{2}}-w^{2} \nabla^{2} T+\frac{w^{2}}{\lambda} p
$$

At present some experimental works are available which confirm a wave nature of heat transfer [7].

A geometrical approach involving Riemannian manifolds to composition of differential equations, particularly those 